\title{
Study on Detection and Classification of Tetracycline Residue in Duck Meat Using Synchronous Fluorescence Spectra and Support Vector Machine
}

\author{
Haibin Xiao, Muhua Liu, Haichao Yuan, Qian Hong and Jinhui Zhao*
}

Optics-Electrics Application of Biomaterials Lab, College of Engineering, Jiangxi Agricultural University, Nanchang China 330045

\begin{abstract}
To the rapid detection of whether the tetracycline residues are excess in duck meat, the optimum characteristic wavelength difference $\lambda$ was determined by synchronous fluorescence analytical method. The recognition model of different residual levels of tetracycline was established by using support vector machine classification algorithm. Firstly, the optimum wavelength difference $\lambda$ for duck meat samples was determined as $70 \mathrm{~nm}$, and synchronous fluorescence spectra of different samples under the condition of $\lambda 70 \mathrm{~nm}$ were collected. Secondly, original synchronous fluorescence spectra were preprocessed by using standard normal variables change (SNV). Finally, 18 wavelength variables were selected from 121 wavelength variables of pretreatment spectra by using competitive adaptive reweighted sampling (CARS). Then the radial basis function (RBF) was selected as the kernel function of support vector classification (SVC), and the optimal kernel function factor $\mathrm{C}$ and $\mathrm{g}$ were determined as 2.83 and 1 , respectively, which were obtained by using grid searching combined with 5-fold cross validation. The classification model of SNV-CARS-SVC was established, and the classification accuracy rate of the model was $95.7 \%$ for prediction sets samples. The results showed that the synchronous fluorescence analysis method could identify tetracycline different residual levels quickly and accurately, and a feasible method was provided for identifying the quality of duck meat.
\end{abstract}

Keywords: synchronous fluorescence spectra, competitive adaptive reweighted sampling, support vector machine, tetracycline, duck meat.

\section{Introduction}

Duck meat is rich in high protein, low cholesterol and low fat. Generally, duck meat contains more vitamin B, vitamin $\mathrm{E}$ and nicotine acid than other meat products, and has rich nutritional value of nourishing stomach and reinforcing kidney [1]. Tetracycline, which is a kind of broad-spectrum sterilization and antibacterial antibiotics, has been widely used as feed additives for promoting growth and keeping health, and are also used to prevent and treat infectious diseases because of low cost, strong sterilization ability and wide antibacterial [2]. A series of problems has emerged as a result of such extensive applications in animal industry, for example a large number of tetracycline residues were found in poultry products. Furthermore, the tetracycline residues can affect meat quality and endanger human body health through the food chain.

Conventional detection methods of tetracycline in poultry products include microbiological method [3], HPLC [4], spectrophotometry [5], TLC [6] and etc. For example, Yantu Zhang et al [7] used high performance liquid chromatography with chemiluminescence to detect tetracycline residues in milk. Although the methods are sensitive and have low limits of detection, they are time-

*E-mail address: zjhxiaocao@sina.com

ISSN: 1791-2377 @ 2013 Kavala Institute of Technology. All rights reserved. consuming, and can't achieve requires of rapid detection because of the complicated and expensive sample preparation procedures. Xiaorong Huang et al [8] developed a method based on microorganism for detecting antibiotics residues in food, but the enrichment process was timeconsuming. These conventional methods tend to timeconsume and complicate sample preparation processes. In general, it was difficult to achieve requirements of rapidly detecting whether tetracycline residues were excess in duck meat. However, the application of tetracycline residues in duck meat by synchronous fluorescence was rarely reported. Synchronous fluorescence spectrometry [9], [10], [11] is a simple method for the detection of complex background interference analytes, and can narrow band and reduce the scattering interference. Therefore, a recognition model, which was used to distinguish whether tetracycline residues were excess in duck meat, was established by using synchronous fluorescence spectrometry [12] combined with SVM classification algorithm in this paper. Synchronous fluorescence spectra of 70 duck samples containing different concentrations of tetracycline were measured using solid collection device of the Cary Eclipase instrument. The characteristic variables for the spectra of the tetracycline in duck meat were selected by using CARS [13], [14]. Finally, SVM [9], [15] was used to build the classification model in order to achieve the goal of rapidly identifying the quality of duck meat. 


\section{Materials and Methods}

\subsection{Reagents and Instruments}

Duck meat was purchased from the vegetable market of Jiangxi Agricultural University; Tetracycline (98.0\%) was obtained from standard substances network of china; Sodium hydroxide; Ultrapure water (Kertone Co., Ltd.) was used for the preparation of all aqueous solutions. Unless stated otherwise, all chemicals were of analytical reagent grade and used without further purification.

Fluorescence spectra were recorded using Cary Eclipase spectrofluorophotometer (Varian, USA) with the solid collection device; JK-50B ultrasonic cleaner was purchased from Hefei Jinnike Machinery Co., Ltd; FA1004B electronic weigher (accuracy $0.1 \mathrm{mg}$ ) was purchased from Shanghai Shangping instrument Co., Ltd; FD-1-50 vacuum freezedrying machine (Beijing Boyikang instrument Co., Ltd); HH-6 digital thermostat water bath (Shanghai Pudong physical optical Instrument Factory).

\subsection{Preparations of Samples}

(1)The breast meats were removed from ducks and the membranes were removed from the breast meats, they were frozen for a moment in the refrigerator. The breast meats were cut into thin slices, and then frozen for some time. (2)Water was removed from the breast meats of frozen by vacuum freeze dryer for 30 hours, and 70 circular samples were made into the diameter of $1.3 \mathrm{~cm}$ and the thickness of $2 \mathrm{~mm}$. (3) $500 \mathrm{mg} / \mathrm{L}$ tetracycline solution was prepared by dissolving $100 \mathrm{mg}$ of standard substance in $200 \mathrm{~mL}$ ultrapure water, working solutions were freshly prepared from stock solution by appropriate dilution with ultrapure water. (4) $1.5 \mathrm{~mol} / \mathrm{L} \mathrm{NaOH}$ solution was prepared by dissolving $3 \mathrm{~g}$ of sodium hydroxide in $50 \mathrm{~mL}$ volumetric flask with ultrapure water. (5) Different concentrations of tetracycline solution were added into 70 brown volumetric flasks of 10 $\mathrm{mL}$, meanwhile, the same volume of $\mathrm{NaOH}$ solution was added. And the mixed solution was degraded in boiling water for 30min. (6) Duck samples were soaked into the different concentrations of tetracycline degradation solution for half an hour, 70 duck samples containing different concentrations of tetracycline were obtained after they were dried on the condition of the room temperature. (7)The synchronous fluorescence spectra of duck samples were collected by using spectrophotometer with the solids collection device, parameters were set as follows: The wavelength difference $\lambda$ was $20 \sim 120 \mathrm{~nm}$, and the interval was $5 \mathrm{~nm}$; The voltage was set at $680 \mathrm{~V}$; Excitation and emission slits were set at $10 \mathrm{~nm}$ and $5 \mathrm{~nm}$, respectively; Emission filter was set at $295 \sim 1100 \mathrm{~nm}$; The spectra was scanned with middle scanning speed in the wavelength range of $240 \sim 360 \mathrm{~nm}$.

In the production process of the samples, the tetracycline content of each sample was obtained by the analysis, and the concentration range was $0.081 \mathrm{mg} / \mathrm{kg} \sim 180.7 \mathrm{mg} / \mathrm{kg}$. The detection sensitivity was slightly lower than the solution state because the spectra of the samples were directly detected. 11 samples of the concentration range between $0.081 \mathrm{mg} / \mathrm{kg}$ and $3.2 \mathrm{mg} / \mathrm{kg}$ were classified as low degree of residues. 27 samples of the concentration range between $6.9 \mathrm{mg} / \mathrm{kg}$ and $95.9 \mathrm{mg} / \mathrm{kg}$ were classified as moderate residues. 32 samples of the concentration range between $104.2 \mathrm{mg} / \mathrm{kg}$ and $180.7 \mathrm{mg} / \mathrm{kg}$ were classified as higher degree of residues. The relevant data is shown in Table 1.

\subsection{Analysis Methods of Data}

Firstly, the preprocessing of spectra was considered in the classification model whether the tetracycline residues are excess in duck meat. The performances of four preprocessing methods, which were multiplicative scatter correction (MSC), smooth handling, standardized processing and standard normal variable transformation (SNV), respectively, were compared, and the appropriate method of pretreatment was determined through the level of classification accuracy of the classification model. Then, synchronous fluorescence spectra of 70 samples were preprocessed by the optimal pretreatment method. The characteristic wavelength variables of tetracycline were selected by CARS, and the algorithm principles and methods reference [13], [14]. Finally, the selected variables were used as the input variables of the SVM classification algorithm, and the recognition model of tetracycline residues levels was established. The best parameters of SVM classifier were obtained by using grid searching combined with the 5-fold cross validation method. According to the principle of division of $2: 1,47$ samples were used to train SVM classifier, and the remaining 23 samples were used to predict. The relevant procedures were completed by MATLAB 2010b (The Math Works, USA) software.

Table 1: Statistical result of tetracycline of duck meat in data sets

\begin{tabular}{ccccc}
\hline Classes & $\begin{array}{c}\text { Number } \\
\text { of } \\
\text { samples }\end{array}$ & $\begin{array}{c}\text { Minim- } \\
\mathrm{mg} / \mathrm{kg}\end{array}$ & $\begin{array}{c}\text { Maxim- } \\
\mathrm{um} / \mathrm{kg}\end{array}$ & $\begin{array}{c}\text { Average } \\
\mathrm{mg} / \mathrm{kg}\end{array}$ \\
\hline $\begin{array}{c}\text { low degree } \\
\text { of residues } \\
\text { moderate } \\
\text { residues } \\
\text { higher }\end{array}$ & 11 & 0.08 & 3.20 & 1.59 \\
$\begin{array}{c}\text { degree of } \\
\text { residues }\end{array}$ & 32 & 104.2 & 180.7 & 138.6 \\
\hline
\end{tabular}

\section{Results and discussion}

\subsection{Synchronous Fluorescence Spectra of Samples}

As shown in Fig. 1, the 3D synchronous fluorescence spectra were scanned for duck meat samples containing tetracycline. In most of synchronous fluorescence studies for different systems, the selection of wavelength difference $\lambda$ is very important. The influence of $\lambda$ can be substantial on the spectral shape, width, signal strength and spectral degree of overlap. In this paper, in order to obtain the best value of

$\lambda$, 3D synchronous fluorescence spectra were studied between $20 \mathrm{~nm}$ and $120 \mathrm{~nm}$, and three-dimensional data was made up of the fluorescence intensity, excitation wavelength and the wavelength difference $(\lambda)$. It can be observed that the fluorescence peaks of the samples, as shown in Fig.1, the duck meat has background fluorescence peaks at the excitation wavelength of about $290 \mathrm{~nm}$. And shown in Fig.1 (b), the background fluorescence peaks of duck meat and tetracycline peaks were well separated, and the fluorescence peak of tetracycline in duck meat was located the excitation wavelength of about $334 \mathrm{~nm}$.

Three dimensional synchronous fluorescence spectra contained the abundance of characteristic peaks, and scanning of the spectra was time-consuming, thus, that is not conducive to rapidly analysis. According to the analysis, the results showed that the signal intensity of fluorescence peaks was higher when the wavelength difference $\lambda$ was equal to 
$70 \mathrm{~nm}$. The synchronous fluorescence spectra of 70 samples were selected on the condition of $\lambda 70 \mathrm{~nm}$ for data analysis and processing.
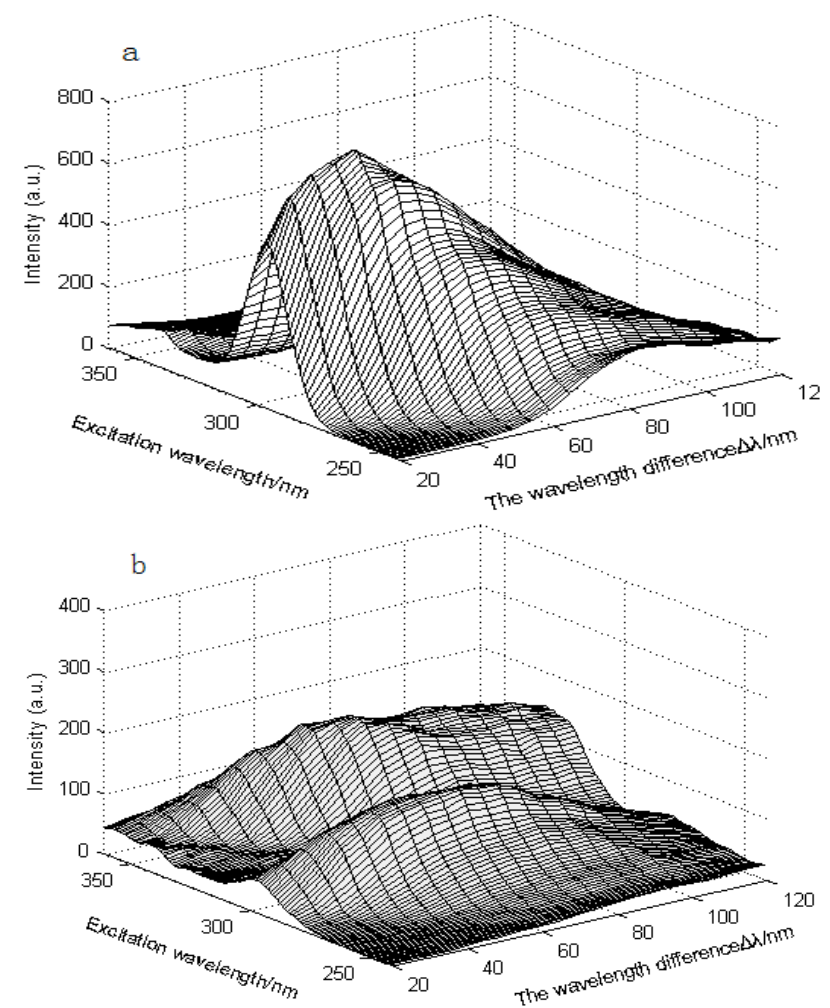

Fig.1. The 3D synchronous fluorescence spectroscopy of duck meat (a) and duck meat containing tetracycline (b)

\subsection{Preprocessing of Synchronous Fluorescence Spectra}

The information of synchronous fluorescence spectra not only contained the substance signals, but also the noise which was caused the instrument or the environment. In order to minimize error and improve the classification accuracy of SVM classifier, the original synchronous fluorescence spectra of samples were pretreated by using MSC, Smooth, Normalization and SNV, respectively, and the four pretreatment methods had a different impact on the classification forecast model.

Table 2: Results of CARS-SVC model with different pretreatment methods

\begin{tabular}{ccc}
\hline $\begin{array}{c}\text { Modeling } \\
\text { methods }\end{array}$ & $\begin{array}{c}\text { Number of correct } \\
\text { classify /prediction } \\
\text { samples }\end{array}$ & $\begin{array}{c}\text { Classification } \\
\text { accuracy\% }\end{array}$ \\
\hline $\begin{array}{c}\text { SNV-CARS- } \\
\text { SVC }\end{array}$ & $22 / 23$ & 95.7 \\
$\begin{array}{c}\text { MSC-CARS- } \\
\text { SVC }\end{array}$ & $21 / 23$ & 91.3 \\
$\begin{array}{c}\text { Smooth- } \\
\text { CARS-SVC }\end{array}$ & $21 / 23$ & 91.3 \\
$\begin{array}{c}\text { Normalization } \\
\text {-CARS-SVC }\end{array}$ & $21 / 23$ & \\
$\begin{array}{c}\text { None-CARS- } \\
\text { SVC }\end{array}$ & $19 / 23$ & 91.3 \\
\hline
\end{tabular}

The characteristic variables associated with tetracycline, which were selected from spectrum pretreatment data by CARS, were determined as the inputs of SVM classifier.
Different classification results of tetracycline residual level in duck meat were shown in Table 2. The model was built up by CARS-SVC. The highest classification accuracy of the CARS-SVC model was $95.7 \%$ after the spectra were preprocessed by SNV pretreatment method. The other three spectra preprocessing classification accuracy were all $91.3 \%$, and the spectra of none preprocessing classification accuracy was lowest, only $82.6 \%$. Therefore, the SNV were selected for spectra pretreatment method in subsequent data processing and analysing

\subsection{CARS Variable Selection Method}

The SVC model was taken more time to calculate, and would affect the classification accuracy because the full band spectra contain a large number of irrelevant information and background noise signal. Characteristic variables were selected from the full band spectra before modeling. In this paper, a new variable selection method called CARS [13] was used to collect informative variables and remove unimportant variables. The method imitated the principle of "survival of the fittest", each wavelength of the spectrum as an individual. Major wavelength points of the PLS model were selected by using adaptive re-weighted sampling technique, and small weight wavelength points were removed. Thesubset with the lowest root mean square error of cross-validation was considered as the best variable subset. The sampling number was 50, and the PLS crossvalidation was 5-fold. The classification model was established when 18 wavelength variables were selected by CARS as the inputs of the model. As shown in table 3, the CARS method was used to extract wavelength variables, the model was not only simple and quick, but also classification accuracy increased to $95.7 \%$ from $87.0 \%$.

\begin{tabular}{ccc}
\multicolumn{3}{c}{ Table 3: Results of SVC model using CARS methods } \\
\hline $\begin{array}{c}\text { Modeling } \\
\text { methods }\end{array}$ & $\begin{array}{c}\text { Number of correct classify } \\
\text { /prediction samples }\end{array}$ & $\begin{array}{c}\text { Classification } \\
\text { accuracy } \%\end{array}$ \\
\hline $\begin{array}{c}\text { SNV-SVC } \\
\text { SNV-CARS- }\end{array}$ & $20 / 23$ & 87.0 \\
SVC & $22 / 23$ & 95.7 \\
\hline
\end{tabular}

\subsection{SVC Model}

Support vector machines (SVM) [9] based on the structural risk minimization principle. It can provide good generalization performance in the classification problem, and have the characteristics of versatility and robustness. Solving ability of model is reflected in the small sample size, the local minimum point and non-linear problem. SVM [15] can obtain the optimal classification results in the linear separable problems. The nonlinear separable problem can map to be divided into high-dimensional space by a nonlinear mapping. An inner product function was defined as non-linear mapping that was the kernel function. Radial basis function selected can help reduce the complexity of training process of classification model, because the residual tetracycline samples belong to the non-linear separable problems.

Kernel function types and the choice of the relevant parameters (penalty parameter $\mathrm{C}$ and kernel function parameters g) were important for the SVM classification model. The $C$ parameters are related to the size of the error penalty score, $g$ parameters are related to the function of the regression error and the parameters even affect the initial value of the feature vector and the characteristic value. Grid 
search combined with 5-fold cross-validation were usually employed to find the optimal parameters of the model for the training samples. The process of optimization parameters was as follows: Firstly, the kernel parameters $\mathrm{C}$ and $\mathrm{g}$ were rough chosen, the initial value, the step, the end values were set at $\left[\begin{array}{lll}-10 & 0.8 & 10\end{array}\right]$. Then, the parameters $(C, g)$ of each pair of values were searched by using the grid search method, the best parameters were found by using the 5-fold crossvalidation method which could make the cross-validation accuracy rate highest. The parameters were carefully chosen based on the results of a rough choice. The initial value, the step, the end values of $\mathrm{C}$ and $\mathrm{g}$ were changed at [-2 0.54$]$ and $\left[\begin{array}{lll}-4 & 0.5 & 4\end{array}\right]$, respectively. And the best parameter pairs (C\&g) were selected. As shown in Fig.2, a rough selection and choiceness search results of the figure were obtained, the abscissa and ordinate expressed the $\log 2 \mathrm{C}$ and $\log 2 \mathrm{~g}$ of parameters, respectively. A contour plot of cross-validation accuracy of the SVM classification model was described in Fig.2. It was shown in Fig. 2 that the cross-validation of the model prediction accuracy rate was up to $82.98 \%$ at $\mathrm{C}=2.83$ and $\mathrm{g}=1$.
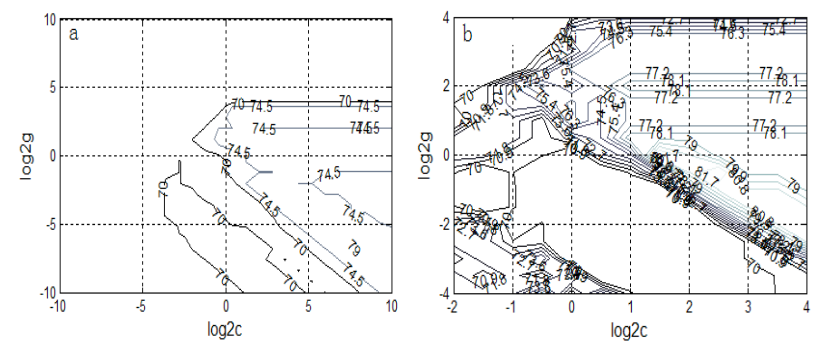

Fig.2. The contour plot of cross-validation prediction accuracy rate of SVM model by roughing (a) and choiceness (b)

\subsection{Prediction Results of SVC Model}

The $\mathrm{C}=2.83$ and $\mathrm{g}=1$ as the best parameters of the kernel function RBF were found by using grid search combined with 5-fold cross-validation method, and the SVM classifier was trained for training samples, then, SVC model of tetracycline residues in different levels was established. The classification accuracy rate was $95.7 \%$ for prediction samples. The plot of actual classes and predictive classes of SVC model was shown in Fig.3. A sample of the second class was classified as the third class mistakenly, and other samples were predicted correctly.

\section{Conclusion}

The wavelength difference $\lambda=70 \mathrm{~nm}$ was selected by analyzing three dimensional synchronous fluorescence spectra of duck samples containing tetracycline. 18 wavelength variables related to tetracycline were selected by using CARS from synchronous fluorescence spectra after SNV spectral preprocessing. Variable numbers were reduced from 121 to 18 , and the compression rate was $85.1 \%$. SVC was employed to establish classification model of tetracycline residual level in duck meat, the classification accuracy of SNV-CARS-SVC prediction model was $95.7 \%$. A new method was provided for the rapid and accurate detection of whether the tetracycline residues are excess in duck meat.

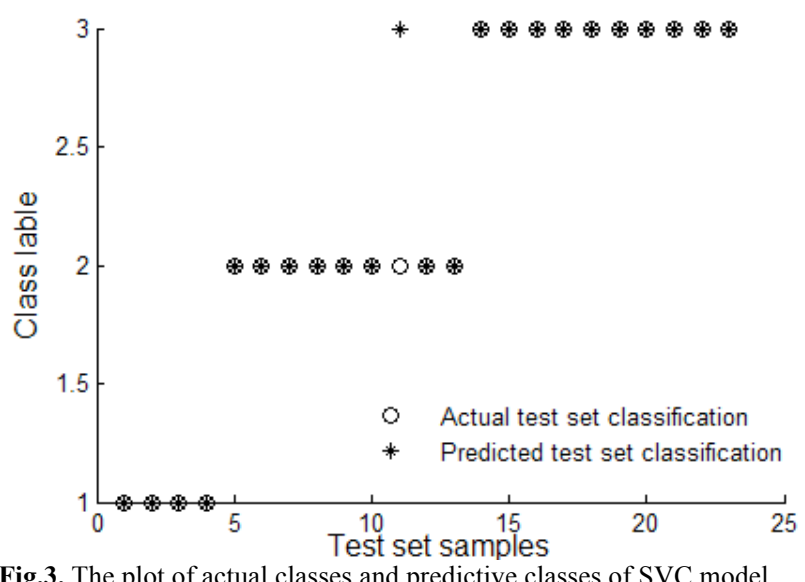

Fig.3. The plot of actual classes and predictive classes of SVC model

\section{Acknowledgments}

This research was supported by National Natural Science Foundation of China (31101295) and National Key Technology R\&D Program (2012BAK17B02). Additional support for this research was provided by External Science and Technology Cooperation Plan of Jiangxi Province, China (20132BDH80005) and the Science and Technology Support Project of Jiangxi Province, China (2012BBG70058) and the Science and Technology Project of Education Department of Jiangxi Province, China (GJJ12244).

\section{References}

1. Wang, W., Zha F.B., Zhang, J., Shen, J.C., and Ren Z.W, "Optimization of SPME-GC-MS Conditions for Flvour Compounds Determinationin in Duck Meat by Response Surface Analysis", Food Science 31(20), 2010, pp. 329-333.

2. Wang, H.Z., Luo, Y., Xu, W.Q., Zhou, Q.X., Tang, B.H. and Wang Y.Y, "Ecotoxic Effects of Tetracycline and Chlortetracycline on Aquatic Organisms", Journal of AgroEnvironment Science 27(4), 2008, pp. 1536-1539.

3. Fagbamila I., Kabir J., Abdu P., Omeiza G., Ankeli P., Ngulukun S., Muhammad M. and Umoh J., "Antimicrobial Screening of Commercial Eggs and Determination of Tetracycline Residue Using Two Microbiological Methods", International Journal of Poultry Science 9(10), 2010, pp. 959-962.

4. Evaggelia, N. E. and Victoria, F. S., "Confirmatory development and validation of HPLC-DADmethod for the determination of tetracyclines in gilthead seabream (Sparus aurata) muscle tissue", Journal of Separation Science 35(10-11), 2012, pp. 1372-1378.
5. Ni, Y.N., Deng, N. and Zhou M, "Kinetic Spectrophotometric Determination of Tetracycline in Pharmaceutical Preparation Samples", Journal of Nanchang University 34(2), 2010, pp. 142146.

6. Elizabeta P., Renata S. R. and Vesna R., "Determination of Tetracycline, Oxytetracycline and Chlortetracycline in Milk by TLC and Column Chromatography Using Am berlite XAD-2", Chemia Analityczna 51(2), 2006, pp. 275-283.

7. Zhang, Y.T., Zhang, Z.J. and Sun, Y.H, "Determination of Tetracyclines Residues in Milk Using High Per-formance Liquid Chromatography with Chemiluminescence Detection", ACTA CHIMICA SINICA 64(24), 2006, pp. 2461-2466.

8. Huang, X.R., Zheng, J., Wu, Q., Chen, B. and Tang, M.Y, "Study on MIA Method for Determination of Antibiotics Residues in Food", FOOD SCIENCE 28(8), 2007, pp. 418-421. 
9. Sun, Y.H., An, H.Y., Jia, X.L. and Wang, J, "Rapid identification of hogwash oil by using synchronous fluorescence spectroscopy", Spectroscopy and Spectral Analysis 32(10), 2012, pp. 2726-2729.

10. Song, G.Q., Xi, H.B., Zhou, Y.X., Li J., Zhao, J.T. and Cui J.H, "Water using constant wavelength synchronous fluorescence spectrometry", Spectroscopy and Spectral Analysis 32(7), 2012, pp. 1838-1841.

11. Yuan, H.C., Zhao, J.H., Liu, M.H., Xiao, H.B., and Xu, J, "Determination of aureomycin content in duck meat white with derivative synchronous fluorescence method", Acta Agriculturae Universitatis Jiangxiensis 35 (1), 2013, pp. 189-194.

12. Zhao, J.H., Yuan, H.C, Liu, M.H., Xu, J. and Xiao, H.B, "Rapid Determination of Neomycin Content in Duck Egg White Using Derivative Synchronous Fluorescence-Wavelet-Subsection Genetic Algorithm-Least Squares Support Vector Regression", Chinese Journal of Analytical Chemistry 41(4), 2013, pp. $546 \sim 552$.
13. Wu, J.Z. and Xu, Y, "NIR Quantitative model optimization of fatty acid in edible oil based on CARS-PLS", Journal of agricultural machinery 42(10), 2011, pp. 162-166.

14. Zhang, H.X., Li, X.N., Fan, W., Liang, Y.Z. and Tang, Y.L, "Determination of Protein and Fat in Liquid Milk by NIR Combined with CARS Variables Screening Method", Journal of Instrumental Analysis 29(5), 2010, pp. 430-434.

15. Zhang, L., Zhang, Y.Z., Wang, Y.K., Zhu, L., Yu F., Zhang, G., Liu, Y. and Wang, A, "Classification of human skin autofluorescence spectrum based on support vector machines for diagnosis diabetes", ACTA LASER BIOLOGY SINICA 21(1), 2012, pp. 65-70 